common on feet infected with Trichophyton spp., presumably because the latter produce antibiotics.

G.Colman

\title{
Pathobiology: how disease happens
}

By Frances K. Widmann. 1978. Boston, Mass.: Little Brown and Co. Pp. xi and 401. £6.90.

At the outset I must say that I enjoyed reading this book though from time to time I had some doubts as to the precise audience at which it has been aimed. The author refers in her preface to "students in the health professions". Is this 'jargonese' for medical and dental students or does the term carry broader connotations? This is not without importance because, in some respects, the treatment of certain subjects is too superficial for medical students and, in others, too detailed for paramedical aspirants. Again, in the preface, Dr Widmann states that "a classic course in pathology is a luxury few curriculums can afford". I suspect that she is using the word "classic" in this context in a pejorative sense, and equates it with teaching programmes in which there is a marked emphasis on morphological detail and 'pattern recognition'.

In her text Dr Widmann, working from a firm base in cell biology, makes a praiseworthy attempt to place the basic processes in disease in a frame of reference composed of the physiological systems that help to maintain a steady state and illustrates her thesis with individual case histories - an excellent idea.

As in any book, one always finds points at which to cavil. Amongst these was the omission of comments on the role of sodium retention in the causation of oedema in patients with congestive cardiac failure and on the labelling of auto-immune thyroiditis as an example of a disease mediated by the escape of a normally 'secluded antigen'.

However, the general concept and pattern of this latest attempt to base the teaching of pathology on events rather than on morphology are good and the book is one that can be read with pleasure and profit.

N. WoolF

\section{Contemporary topics in immuniobiology. Volume 2}

Edited by N. L. Warner and M. D. Cooper. 1978. New York: Plenum Press. Pp. xiv and 272. $£ 17 \cdot 32$.

As the title suggests, this book-like others in the series-is concerned with the present state of knowledge on some of the contentious issues in immunology. Most of the authors write in a manner that presupposes that readers already have a working knowledge of immunology. Furthermore they assume that readers will share their keen insight into those problems requiring solution for further progress in basic immunology. Thus, despite the publisher's assurances on the cover, no one should believe that the solution of practical or clinical problems has been uppermost in the minds of any of the contributors. This is a perfectly responsible attitude on their part. Indeed, this series points the need for a parting of the ways between reviewers who write for academic immunologists and those who seek to interpret immunology for clinicians: at least half the material in the book is culled from observations in experimental animals, a point over which the authors are rightly not in the least apologetic; moreover, the narrative relies heavily on long strings of abbreviations which provide a useful shorthand for the initiated and weary leafing through pages for others. As with lengthy Russian novels the reader really needs a dramatis personae to avoid disorientation.

In contrast with earlier volumes in this series, there is no single unifying theme for the subjects included in this volume. However, they can be divided loosely into two groups, those concerned with analysing effector cells in a variety of immune reactions, and those dealing with the synthesis of immunoglobin receptors on lymphoid cells, and the style and detail will appeal only to the professional immunologist who is obliged to absorb such subjects in minute detail. Even for such readers the speed of events in immunology poses difficulties. Commendably a high 
percentage of the references in all the contributions are to papers published in the 12 months before the book appeared. Nonetheless, the introduction of hybridomas trained to produce monoclonal antibodies of precise specificity is already eclipsing much of the exact and painstaking work recorded in this volume of "contemporary topics". There are some chapters that may interest clinicians and pathologists. Immunologists have for many years tried to persuade clinicians than an imbalance in lymphocyte subpopulations underlies human auto-immune diseases. Moretta and his colleagues have introduced assays which they claim are specific for lymphocyte subpopulations that either promote or inhibit immune reactions, and the distribution of these subpopulations in patients with immunodeficiency and auto-immune diseases is allegedly abnormal. The perennial issue of cancer immunity is dealt with by Haskill and others. All pathologists are now aware that no meaningful deductions can be made about resistance to tumours simply by assessing the amount of "mononuclear cell infiltration" in histological preparations. This contribution reviews the techniques for isolating and characterising the cells infiltrating experimental and human tumours and for assaying their functions in terms of immune reactions to tumours but using specialised terminology. Finally, the subjects of "natural killer cells" has burgeoned into an independent science for those interested in host resistance to tumours and infective agents, yet the subject has scarcely been noticed by clinicians or pathologists. Kiessling and Haller review the evidence for cells in the mouse that kill tumour cells or infected cells through mechanisms that do not involve specific immunological instruction. This chapter like the others cited poses a real dilemma for busy doctors. Some subjects such as the regulation of immunoglobulin synthesis are being investigated so successfully and so intensively that there is an argument for awaiting a nice, clean solution. Other subjects such as the role of "natural killer cells" are still so speculative and contentious that they may never prove clinically relevant. Thus, this book can be recommended only to readers who enjoy the cut and thrust of immunology in the making or to those whose professional preoccupations are encompassed by one or more of the chapters.

A. M. Denman 